\title{
Use of Mycobacterial Culture for the Diagnosis of Smear Negative TB Cases Among New Outpatients at NIDCH, Dhaka
}

\author{
S M Mostofa Kamal ${ }^{1}$, Asif Mujtaba Mahmud ${ }^{1}$, Chowdhury Rafiqul Ahsan ${ }^{2}$, Md Rafiqul Islam ${ }^{1}$, Golam Sarwarl, Md Mostafizur \\ Rahman', Provat Chandra Barua ${ }^{3}$
}

${ }^{1}$ National Institute of Diseases of Chest and Hospital( NIDCH $)^{2}$ University of Dhaka 3 NTP, DGHS.

\begin{abstract}
In this prospective study out of 300 randomly selected clinically and/or radiologically suspected TB patients ultimately data of 277 cases were analyzed. Among 23 excluded cases, 6 were positive by microscopy, 5 responded with broad spectrum antibiotics, and 12 were contaminated. After 2 months follow up with anti TB drugs, a total of $124 \mathrm{~TB}$ cases were diagnosed of which $121(44 \%)$ were culture positive. Three cases were diagnosed by empirical anti TB treatment. A total of 153 cases were true negative by mycobacterial culture. Of 121 isolates 119 were typical and 2 were atypical mycobacteria. In this group, the sensitivity, specificity, positive predictive value and negative predictive value of mycobacterial culture were $97.63 \%, 100 \%, 100 \%$ and $98.1 \%$ respectively. Among the culture proven cases; $79 \%$ were prevalent in male and $21 \%$ in female. Case positivity was significantly higher among patient having symptoms of cough with hemophtysis with or without fever(58\%) than the patients with constitutional symptoms. No advanced or cavitary lesions were seen on X-ray among culture proven cases. Majority culture proven cases(72\%) had minimal lesion on X-ray. After two months anti TB therapy 119 culture proven cases and three culture negative cases improved clinically and radiologically.
\end{abstract}

\section{Introduction:}

National Institute of Diseases of Chest and Hospital( NIDCH), Bangladesh is a tertiary level hospital specially designed to treat difficult TB cases like smear negative cases, MDR-TB cases, chronic and relapse cases and also extra pulmonary TB cases. 16\% of TB cases are new smear negative cases $^{2}$. A good number of these patients are being referred to NIDCH from all over Bangladesh for better management. Traditionally, they are empirically treated with anti TB drugs after having a presumptive diagnosis with ancient practice of positive X-ray and Mantoux test (MT) finding and high ESR. Although a good number of patients of this group are not really TB cases ${ }^{5}$. Besides technical errors of smear negativity, many medical conditions like

\section{Correspondence:}

Dr S M Mostofa Kamal

Assistant Professor, Pathology \& Microbiology

National Institute of Diseases of Chest and Hospital( NIDCH)

Mohakhali, Dhaka-1212, Bangladesh. Tel: +(880)1819401900

e-mail: ntrlnidchbd@yahoo.com resistant bacterial infections, viral infections, carcinoma, lymphoma, asthma, chronic obstructive lung diseases(COPD) other allergic condition came to present as smear negative TB cases $^{6}$. HIV infection also responsible to produce higher number of smear negative TB cases $^{7,8}$. In a high burden country like Bangladesh, microscopy remains as gold standard but mycobacterial culture is superior to microscopy. It can detect cases with bacterial load up to 10 to 100 viable bacilli/ $\mathrm{ml}$ of sputum whereas microscopy can do it up to 5000 to 10000 bacilli/ml. Moreover microscopy can not detect viability, identity and anti TB drugs susceptibility of TB bacilli9,10,11. For bacteriological evidence of smear negative TB cases culture is the next option to microscopy ${ }^{6}$. Unlike developed countries, the infectivity and mortality of smear negative TB cases is the same as that of smear positive TB cases in high burden countries ${ }^{12}$. Moreover, empirical TB treatment may give rise to chronic and MDR cases. Gradual expansion of culture laboratories and introduction of molecular diagnostics can help proper management of TB cases. In spite of high prevalence of smear negative TB cases there are only two review articles regarding this subject in 
last 20 years worldwide ${ }^{6}$. Until now there is no such study in Bangladesh. Therefore, this study will describe the case positivity and natural history of new smear negative TB cases by mycobacterial culture.

\section{M atrials and M ethods:}

A total of 300 randomly selected patients having clinical and/or radiological features of tuberculosis attending out patients department of NIDCH were enrolled in this study during May to October-2008. Smear positive cases or the cases taking prior anti-TB drugs were excluded from the study. One spot sputum specimen (about $5 \mathrm{ml}$ )and another morning specimen were collected under the guidance of a doctor. Both the specimens were sent to laboratory as soon as possible. Two direct smears were prepared from each of the two specimens for staining - one by Ziehl-Nelsen(Z-N) and the other by Flurochrome(FM) techniques and were examined under microscope following standard operating procedure (SOP). If any cases found positive, then it was excluded from the study.

The remaining part of each specimen was processed for culture by digestion, decontamination and concentration following modified Petroff's method and were inoculated on to two slopes of Lowenstein- Jensen $(L-J)$ media for six weeks. Readings were taken every week for six weeks. The identity of the isolates were made by growth rate, colony morphology, P-nitrobenzoic acid(PNB)susceptibility, catal ase and nitrate reduction tests. Drugs susceptibility tests were not performed.

Later on, all the cases were given a course of broad spectrum antibiotic(Levofloxacin/ciprofloxacin for one week) and were asked to perform a fresh chest X-ray after two weeks. If the patients improved clinically and radiologically they were excluded from the study. Rest of the patients were given anti TB drugs following the national anti - TB treatment guidelines. After two months, clinical and radiological improvement were noted. HIV screening was not performed.

\section{R esults:}

Among 121 culture proven cases $79 \%$ were male and $21 \%$ were female. The culture positivity were more prevalent in the age group 41 years or above (Tablel)
Table I: Age sex distribution of culture positive T B cases among actual study population

\begin{tabular}{lllll}
\hline Age Group & \multicolumn{2}{c}{$\begin{array}{c}\text { Malen }=201(72.6 \%) \\
\text { Culture }\end{array}$} & \multicolumn{2}{c}{$\begin{array}{c}\text { Femalen =76(27.4\%) } \\
\text { Culture }\end{array}$} \\
& Positive & Negative & Positive & Negative \\
\hline $10-20$ yrs. & 0 & 04 & $02(33.9 \%)$ & $04(67 \%)$ \\
$n=4+6=10$ & & & & \\
$21-30$ yrs. & 28 & 39 & 11 & 21 \\
$n=67+32=99$ & $(42 \%)$ & $(58 \%)$ & $(34 \%)$ & $(66 \%)$ \\
$31-40$ yrs. & 16 & 20 & 06 & 10 \\
$n=36+16=52$ & $(44 \%)$ & $(56 \%)$ & $(37 \%)$ & $(63 \%)$ \\
$41-50$ yrs & 22 & 15 & 05 & 12 \\
$n=37+16=53$ & $(59 \%)$ & $(41 \%)$ & $(25 \%)$ & $(75 \%)$ \\
$>50$ yrs. & 30 & 27 & 2 & 4 \\
$n=57+6=63$ & $(52 \%)$ & $(48 \%)$ & $(33 \%)$ & $(67 \%)$ \\
Total $n=277$ & $96(79 \%)$ & 105 & $25(21 \%)$ & 51 \\
Total culture positivecases: $121(44 \%)$ & & \\
\hline
\end{tabular}

Out of 300 patients, 6 were smear positive, 5 responded with broad spectrum antibi otics and 12 were contaminated and all three groups were excluded. 277 (92.3\%) patients comprised the actual study population ( Tablell) .

\section{Table II : C ulture Results}

\begin{tabular}{lll}
\hline Characters & Number & $\%$ \\
\hline Total study population & 300 & 100 \\
Case excluded & & \\
a) smear positive & $06 *$ & 2.0 \\
b) antibiotic responder & 05 & 1.7 \\
c) contamination & 12 & 4.0 \\
$\quad$ Total & 23 & 7.7 \\
Actual study population & 277 & 92.3 \\
\hline
\end{tabular}

*2 by only FM, 4 by both FM \& Z-N methods

121 (44\%) of the remaining 277 were culture positive and the rest of the 156 patients were culture negative cases (table III).

Table III : Distribution of Culture positive and negative cases among the actual study population

\begin{tabular}{lll}
\hline $\begin{array}{l}\text { Actual study population } \\
(\mathrm{n}=277)\end{array}$ & Total number & Percentage \\
\hline Culture positive & 121 & $44 \%$ \\
Culture negative & 56 & $56 \%$
\end{tabular}

In addition to 121 culture positive cases, 3 patients of the 156 culture negative cases improved by anti TB drugs and the 
remaining 153 were consi dered as true negative cases (TableIV) .

Table IV : Distribution of cases considered as T B

\begin{tabular}{lll}
\hline & Total No & Percentage \\
\hline $\begin{array}{l}\text { Number of study subjects } \\
\text { that were culture positive }\end{array}$ & 121 & 97.6 \\
$\begin{array}{l}\text { Number of study subjects } \\
\text { that were culture negative } \\
\text { but improved with anti TB } \\
\text { treatment }\end{array}$ & 03 & 2.4 \\
Total number of TB cases & 124 & 100 \\
\hline
\end{tabular}

Among 121 isolates, 119 were typical mycobacteria and 2 atypical mycobacteria (TableV ).

\section{Table V: Identity of I solates}

\begin{tabular}{lll}
\hline Identity & No & Percentage \\
\hline Mycobacterium tubercul osis & 19 & 99.30 \\
Atypical Mycobacteria & 02 & 0.7 \\
Total & 121 & 100 \\
\hline
\end{tabular}

Of the 121 culture positive cases, 27 presented with only cough, 49 cough with low grade fever, 24 cough with weight loss, 14 cough, hemoptysis with or without fever only 7 with cough and constitutional symptoms (TableVI)

\section{Table VI : R elation of C linical Finding and C ulture Positive Cases}

\begin{tabular}{|c|c|c|c|}
\hline \multirow[t]{2}{*}{ Clinical findings* } & \multicolumn{3}{|c|}{ Total patients Culture Positive } \\
\hline & no (\%) & No & \%of total case \\
\hline Only Cough & $69(25 \%)$ & 27 & 39 \\
\hline Cough with low & $92(33 \%)$ & 49 & 48 \\
\hline Grade fever & & & \\
\hline Cough with weight loss & $56(20 \%)$ & 24 & 43 \\
\hline $\begin{array}{l}\text { Cough, hemophtysis } \\
\text { with or without fever }\end{array}$ & $23(08 \%)$ & 14 & 61 \\
\hline $\begin{array}{l}\text { Cough with constitutional } \\
\text { symptoms }\end{array}$ & $37(13 \%)$ & 07 & 19 \\
\hline Total & $277(100 \%)$ & 121 & 44 \\
\hline
\end{tabular}

*May or may not associated with X-ray findings
There were no advanced or cavitary lesions in X-ray of any patients. However 113 cases had minimal diseases, 6 had moderate non cavitary lesion but only 02 culture positive cases had no shadow on the X-ray ( TableVII) .

Table VII : Relation of X-ray finding and C ulture positive cases

\begin{tabular}{|c|c|c|c|c|}
\hline \multirow{2}{*}{$\begin{array}{l}\text { X-ray } \\
\text { finding }\end{array}$} & \multicolumn{2}{|c|}{ Total no of patients } & \multicolumn{2}{|c|}{ Culture Positive } \\
\hline & No & $\%$ & No & \%of total patients \\
\hline No shadow & 112 & 40 & 02 & 1.8 \\
\hline $\begin{array}{l}\text { Minimal } \\
\text { disease }\end{array}$ & 158 & 57 & 113 & 72 \\
\hline $\begin{array}{l}\text { Moderate } \\
\text { non- cavitary }\end{array}$ & 97 & 2.5 & 06 & 86 \\
\hline $\begin{array}{l}\text { Advanced } \\
\text { cavitary }\end{array}$ & 0 & 0 & 0 & 0 \\
\hline Total & 277 & 100 & 121 & 44 \\
\hline
\end{tabular}

When, culture results were compared to total TB cases diagnosed, the sensitivity , specificity, positive predictive val ue and negative predictive val ue were $97.63 \%, 100 \%, 100 \%$ and $98 \%$ respectively(TableVIII ).

Table VIII : C omparison of culture result among total TB cases

\begin{tabular}{llll}
\hline Culture Result & Positive & $\begin{array}{l}\text { TB cases } \\
\text { Negative }\end{array}$ & Total \\
\hline Positive & 121 & 0 & 121 \\
Negative & 03 & 153 & 156 \\
Total & 124 & 153 & 277 \\
\hline
\end{tabular}

Sensitivity $=97.63 \%$ Specificity $=100 \%$

Positive predictive value (PPV) $=100 \%$ Negative predictive value $=98.1 \%$

\section{Discussion:}

In this study the case positivity rate was $44 \%$ (121 of 277). The remaining $54 \%$ cases were may be due to resistant bacterial infections, viral infection . parasitic infestations, lymphoma, carcinoma, allergic lung conditions and asthma or COPD ${ }^{6}$. Some of them may be due to tubercul osis but at present they are not detectable by culture. The empirical anti TB treatment of negative cases would be over-diagnosed. But culture can eliminate these clinical 
problem in TB case management. There are variable reports regarding culture positivity among smear negative cases. In one review study (Van Deun A.,2004) showed 24 to 62\% positivity rate among different geographical locations ${ }^{3}$. Other natural history of smear negative had similarities with smear positive TB cases$^{2}$.

\section{Conclusion:}

Mycobacterial culture appears as good tool for diagnosis of smear negative TB cases specially in TB/HIV co infections. It can eliminate the problem of over diagnosis of smear negative TB cases with traditional clinical and radiological basis. NTP Bangladesh can adopt policy for case detection among smear negative cases by culture through expanding culture facilities all over the country.

\section{Acknowledgement :}

This study was funded by NTP, DGHS, Bangladesh.

\section{R eferences:}

1 World Health Organization, Geneva, Global Tuberculosis Report: surveillance, planning, financing, 2007.(WHO/HTM/TB/2007.376)

2 NTP/MOHFW \& WHO. Tuberculosis in Bangladesh. Annual Report: 2006

3 Van Deur, A. What is the role of mycobacterial culture in diagnosis and case definition? Toman's Tuberculosis, 2004.WHO, Geneva.(Page: 35- 43)

4 Harris, A. What is the additional yield from repeated sputum examinations by smear microscopy amd cilture?tomans Tubercul osis, 2004. WHO, Geneva.(Page: 46- 50)
5 Harris, A. What are the relative merits of chest radiography a d sputum examination (s,ear microscopy and culture) in case detection among mew outpatients with prolonged chest symptoms? Toman's tubercul osis, 2004. WHO, Geneva.(Page: 61-65)

6 R. Colebunders, I. Bastian. A review of the diagnosis and treatment of smear-negative pulmonary tuberculosis.INT J TUBERC LUNG DIS 4(2): 97-107

7 Murray CJ L, Styblo K, Rouillon A. Tuberculosis in Developing countries: burden intervention and cost. Bull int Union Tuberc Lung dis 1990; 65(1):6-24.

8 De Cock K M, Speo B, Coulibaly I M, Lucas S B. Tubercul osis and HIV infection in sub- Saharan Africa . JAMA 1992; 268: 1581-1587.

9 Editorial. Smear-negative pulmonary tuberculosis . Tubercle 1980; 61: 113-115.

10 Parry C M, Sputum smear negative pulmonary tuberculosis.Tropical Doctor 1993; 23: 145-146

11 KimT C, Blackman R S,Heatwole K M , KimT, Rochester D E Acid-fast bacilli in sputum smears of patients smears of patients with pulmonary tubercul osis. AmRev Respir Dis 1984; 129:264-268.

12 Rouillon A, Perdrizet S, Parrot R, Transmission of tubercle bacilli: the effects of chemotherapy. tubercle 1976; 57: 275299.

13 Harris A D, Maher D, Nunn P.An approach to the problems of diagnosis and treating adult smear-negative pulmonary tuberculosis in high HIV- prevalence setting in sub-Saharan Africa. Bull World health organ 1998;76:651- 662.

14 Hong Kong Chest Service / Tuberculosis Research Centre, Madras/ British Medical Research Council. A study of the characteristics and course of sputum smear- negative pulmonary tubercul osis. Tubercle 1981;62:155-167. 